\title{
Exstensive gastric heterotopia of the distal ileum: A case of bowel obstruction
}

Elisa Maria Vaterlini, Chiara Osio, Giana Panagini, Stefano Grotto, Giuseppe Pelosi, Andrea Ferretti, Marco De Luca, Tagliabue Marta, Davide Marenco, Valerio Ceriani and Salvatore Guarino*

${ }^{*}$ Correspondence: salvatore.guarino@gmail.com

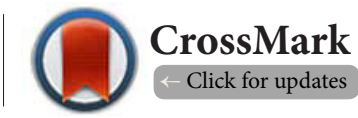

IRCCS Multimedica, Sesto San Giovanni, Italy.

\begin{abstract}
Gastric heterotopia $(\mathrm{GH})$ is a rare congenital condition, than refers to the presence of gastric mucosa in other organs, it can be potentially found in any anatomical sites in the body, but it typically involves the gastrointestinal tract. Herby we report a case of bowel obstruction caused by a long segment of distal ileum affected by GH. The disease had determined complete obliteration of the ileum lumen. Patient was successfully treated with a right hemicolectomy extended to the terminal ileum and primary ileocolic anastomosis. The presence of GH in the small bowel beyond the ligament of Traitz is generally found associated with congenital anomalies like the Meckel's diverticulum or intestinal duplication, while its isolate finding is extremely rare. It is even more rare the presence of a long tract of small bowel affected by GH causing intestinal obstruction. Its diagnosis can be challenging as symptomatology is generally very vague and depends on the site and on the extent of the disease. Its clinical presentation and radiological findings can often mimic Inflammatory Bowel Disease (IBD). GH can present as occult bleeding, abdominal pain, but extremely rarely it cause bowel obstruction.

We believe that GH has to be listed in the differential diagnosis and opportunely investigated in those cases of occult bleedings, abdominal pain and dyspepsia where other diagnosis have been already ruled out.
\end{abstract}

Keywords: Gastric heterotopia, Bowel obstruction

\section{Case Report}

A 88-year-old man was admitted to the Emergency Department with acute onset of abdominal pain associated with vomiting and five days of severe constipation. His past medical history included hypertension and a previous episode of bowel obstruction ten years before, conservatively treated. Upon admission he presented with normal vital signs, abdominal tenderness (Bloomberg's sign positive), and no bowel sounds. His blood tests showed signs of inflammation and dehydration, acute renal failure and increased lactates. The CT abdomen he performed in A\&E revealed small bowel obstruction with a transition point at the level of the distal ileum where a long segment of small bowel was thickened and edematous causing complete obliteration of the lumen. Radiological findings were suggesting obstruction caused by IBD. A naso-gastric tube was inserted and it drained almost 2 liters of enteric content.
Emergency laparotomy was performed: a $50 \mathrm{~cm}$ long segment of distal ileus appeared to be thickened and hard on palpation. We decided to perform right hemicolectomy including the $50 \mathrm{~cm}$ long segment of diseased ileus. Ileocolic primary anastomosis was performed. Post-operative recovery was free of complications. Bowel function was physiologically restored on day 2 after surgery and patient was started on oral intake right after. He was discharged on day 7 after surgery with no postoperative complications.

The histopathology report revealed, within the segment of small bowel excised, extensive presence of gastric heterotopia associated to multiple ulcerations and luminal para-inflammatory stenosis. Resection margin were free from the disease. Immunophenotype resulted positive for MUC1, MUC2, MUC5AC, MUC6, CDX2, beta-catenina, citocheratine 7 e 20 in line with the diagnosis (Figure 1). 


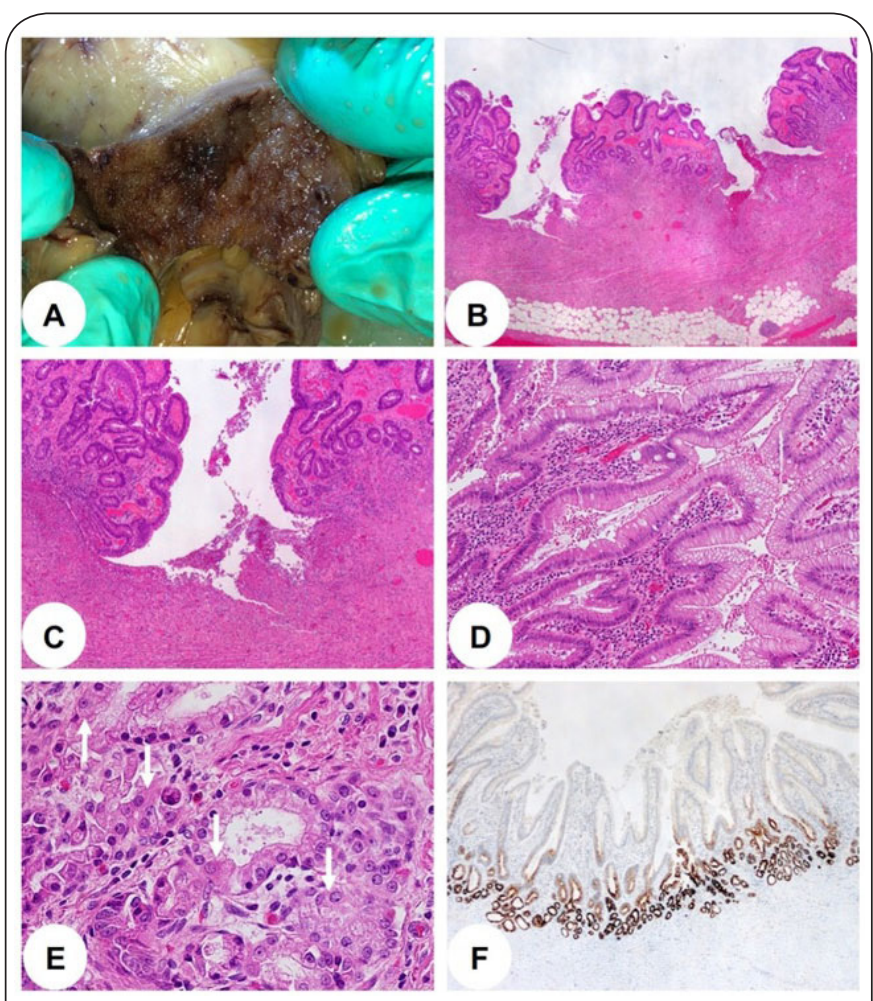

Figure A-F. Gastric heterotopy/ectopy of ileal mucosa. Gross presentation showed an irregular cobblestone appearance of the ileal mucosa surface with linear and serpiginous ulcers delimitating unaffected mucosa islets (A). Microscopically, multiple ulcers were seen, which were separatedfrom each other by unaffected or edematous islets of ileal mucosa (B). Ulcers, however, were superficial and fissures as seen in Crohn disease were absent $(\mathbf{C})$. Intestinal villi were variably covered by gastric-type metaplastic epithelium (D) and in the deep glandular compartment of Lieberkuhn's crypts there were oxyntic-type cells (E, white arrows). Immunohistochemically, strong and diffuse decoration for gastric-type MUC6 (usually lacking in the normal colonic and ileal mucosa) was limited to the deep glandular compartment of ileal mucosa $(\mathbf{F})$.

\section{Discussion}

Heterotopia is defined as the presence of a normal tissue type in non-physiological sites. Gastric heterotopia is defined as the presence of normal gastric mucosa in abnormal locations. It can be found in any anatomical sites, most often in the duodenum, in the esophagus and it is often related with Meckel's diverticulum and intestinal duplication (14). Anectodical cases have been occasionally found in the tongue, rectum, small intestine, gallbladder, and bile duct [1].

$\mathrm{GH}$ has not to be confused with gastric metaplasia (GM). While GM is found in anatomical sites subject to inflammatory processes as in Barret's oesophagus, true heterotopia is not associated to inflammatory process as cause of it and it is more correctly considered an as congenital condition.

$\mathrm{GH}$ is characterized by the presence of full mucosal specialized gastric glands and parietal cells (especially fundic glands) [2]. Diversely gastric metaplasia is defined by the presence of only gastric foveolar epithelium with the absence of specialized gastric glands, and it is caused from repeated inflammatory stimulus to the mucosa [4]. The symptoms caused by gastric heterotopia depends on the location as well as on the extent of the heterotopic tissue. In the majority of cases, the lesions are represented by scanty patches of heterotopic areas within a normal mucosa. These cases can remain asymptomatic and do not cause any medical conditions until they are incidentally diagnosed during endoscopic studies performed for other reasons [4]. Only when the disease reaches a considerable extent within a normal organ, it can become symptomatic. However, in the majority of cases this condition has no specific presentation and no defined imaging or endoscopic features as they mostly depend from the area affected and by its extent. The cases described in the distal esophagus are typically associated with the symptoms of dysphagia, reflux and dyspepsia due to ulcerations or strictures. The localizations within the biliary tract (extremely rare) can cause obstructive jaundice and they are often related with the presence of colangiocarcinoma [10]. Gastric heterotopia found in small and large bowel is associated with nonspecific abdominal pain and it can sometimes cause bowel intussusception, obstruction, ulceration, bleeding, and perforation. These clinical presentations can all mimic IBD.

In the young population, $\mathrm{GH}$ of the small bowel can be a source of gastrointestinal bleeding and can cause iron deficiency anemia, and its diagnosis can be very challenging for the physicians. Accurate past medical history is fundamental, because $\mathrm{GH}$ is often associated with congenital malformations (spina bifida occulta, scoliosis, and malrotation of the colon). Rectal and anal localization of gastric heterotopia has also been reported, often presenting with rectal bleeding or/and strictures. Small bowel location of true gastric heterotopia beyond the ligament of Treitz is rare in the absence of a Meckel's diverticulum. Turck et al. estimated 30 cases reported as of 1990, with a few scattered reports since then [2]. Endoscopy can be helpful in upper Gl localization or in the localization of colon and rectum and terminal ileum, not for the midgut. Endoscopic video capsula may be helpful for finding mucosal anomalies in those tracts that cannot be reaching by endoscopic exams, but it cannot provide biopsy sample for definitive diagnosis nor an exact location [3]. However sometimes it is not easy to identify the lesion because of their locations and features (nonpolypoid or flat morphology). The gastric heterotopic lesions present often as polypoid or nodular aspect. Sometimes the heterotopic epithelium is flat and continues with the normal mucosa, the lesions are circumferential and accentuated folds of mucosa. On microscopic examination, there are "well demarcated zones of gastric mucosa adjacent to normal mucosa" [4]. Matsubara et al reported 28\% of gastric heterotopia harbor GNAS and/or KRAS mutations. Nakagawa et al described that duodenal gastric heterotopia is based upon molecular genetic changes in the Wnt/ $\beta$ - catenin 
Guarino et al., Journal of Histology \& Histopathology 2021,

pathway [4]. These mutations are identified commonly in duodenal adenoma and adenocarcinoma, in fact some authors believe that heterotopia might be a pre-cancerous lesion.

\section{Conclusion}

$\mathrm{GH}$ is a rare condition that could involve every part of the $\mathrm{Gl}$ tract, theoretically also outside the GI tract. It is most frequently in the gastrointestinal tract but it is extremely rare in bowel beyond the ligament of Treitz. It has usually a polypoid/tumoral growth, and rarely a flat pattern of growth.
$\mathrm{GH}$ is often as intomatic or paucisintomatic. Sometimes it can cause serious complications, in relation with the fact that the diagnosis is not easy neither unique, and often delayed: could have devasting sequelae and requires an urgent surgical treatment. We believe this condition has always to be taken in consideration among differential diagnosis of unclear clinical pictures of abdominal pain, bowel obstruction or occult bleeding.

\section{Competing interests}

The authors declare that they have no competing interests.

Authors' contributions

\begin{tabular}{|l|c|c|c|c|c|c|c|c|c|c|c|}
\hline Authors' contributions & EV & CO & GP & SG & GP & AF & MD & TM & DM & VC & SG \\
\hline Research concept and design & $\sqrt{ }$ & -- & -- & -- & -- & -- & $\sqrt{ }$ & -- & -- & $\sqrt{ }$ & $\sqrt{ }$ \\
\hline Collection and/or assembly of data & -- & $\sqrt{ }$ & $\sqrt{ }$ & -- & -- & -- & -- & -- & -- & -- & -- \\
\hline Data analysis and interpretation & -- & -- & -- & -- & -- & -- & -- & $\sqrt{ }$ & $\sqrt{ }$ & -- & -- \\
\hline Writing the article & $\sqrt{ }$ & -- & -- & -- & -- & -- & -- & -- & -- & -- & -- \\
\hline Critical revision of the article & -- & -- & -- & -- & -- & $\sqrt{ }$ & -- & -- & -- & -- & -- \\
\hline Final approval of article & -- & -- & -- & $\sqrt{ }$ & $\sqrt{ }$ & -- & -- & -- & -- & -- & $\sqrt{ }$ \\
\hline Statistical analysis & -- & -- & -- & -- & -- & -- & -- & -- & -- & -- & -- \\
\hline
\end{tabular}

Acknowledgments

This study was supported by the Italian Minister of Health.

Publication history

Editor: Khush Mittal, New York University School of Medicine, USA.

Received: 01-June-2021 Final Revised: 20-Aug-2021

Accepted: 14-Sept-2021 Published: 28-Sept-2021

\section{References}

1. Brahim, E. Ben, Jouini, R., Aboulkacem, S., Jaouadi, F., \&Labbène, N. (2011). Hétérotopiegastrique : Étude anatomo-clinique de 12 cas. 935-939.

2. Lambert, M. P., Heller, D. S., \& Bethel, C. (2000). Extensive gastric heterotopia of the small intestine resulting in massive gastrointestinal bleeding, bowel perforation, and death: Report a case and review of the literature. Pediatric and Developmental Pathology, 3(3), 277-280. https://doi. org/10.1007/s100249910036

3. Sokol, H., Seksik, P., Wendum, D., Bellanger, J., Parc, Y., Cosnes, J., \&Beaugerie, L. (2009). Gastrointestinal bleeding diagnosed using video capsule endoscopy. Gut, 58(9), 2008-2009. https://doi.org/10.1136/ gut.2008.172155

4. Nasir, A., Amateau, S. K., Khan, S., Simpson, R. W., Snover, D. C., \& Amin, K. (2018). The many faces of intestinal tract gastric heterotopia; a series of four cases highlighting clinical and pathological heterogeneity. Human Pathology, 74, 183-187. https://doi.org/10.1016/j.humpath.2018.01.003

5. Salem, G. A., Fazili, J., \& Ali, T. (2017). Gastric heterotopia in the rectum. A rare cause of ectopic gastric tissue. Arab Journal of Gastroenterology, 18(1), 42-43. https://doi.org/10.1016/j.ajg.2017.01.005

6. Alexander, L., Abbas, S., \& Alexander, S. (2018). Symptomatic large ampullary gastric heterotopia. Gastrointestinal Endoscopy, 88(2), 402-403. https://doi.org/10.1016/j.gie.2018.02.031

7. Genta, R. M., Kinsey, R. S., Singhal, A., \&Suterwala, S. (2010). Gastric foveolar metaplasia and gastric heterotopia in the duodenum: No evidence of an etiologic role for Helicobacter pylori. Human Pathology, 41(11), 1593-1600. https://doi.org/10.1016/j.humpath.2010.04.010
8. Srinivasan, R., Loewenstine, H., \&Mayle, J. E. (1999). Sessile polypoid gastric heterotopia of rectum: A report of 2 cases and review of the literature. Archives of Pathology and Laboratory Medicine, 123(3), 222-224. https://doi.org/10.1043/0003-9985(1999)123<0222:SPGHOR>2.0.CO;2

9. Bandi, A., Tan, Y. W., \&Tsang, T. (2014). Correlation of gastric heterotopia and Meckel's diverticular bleeding in children: A unique association. Pediatric Surgery International, 30(3), 313-316.

10. Kim, Y., Jung, M. J., \& Shin, S. J. (2018). Heterotopic Gastric Mucosa in the Common Bile Duct With Cholangiocarcinoma. International Journal of Surgical Pathology, 26(8), 745-748. https://doi.org/10.1177/1066896918 775531s00383-013-3441-2

11. Jimenez, J. C., Emil, S., Steinmetz, B., Romansky, S., \& Weller, M. (2005). Recurrent gastrointestinal tract bleeding secondary to jejunal gastric heterotopia. Journal of Pediatric Surgery, 40(10), 1654-1657. https://doi. org/10.1016/j.jpedsurg.2005.06.010

12. Kumar, R., Tripathi, M., Chandrashekar, N., Agarwala, S., Kumar, A., Dasan, J. B., \& Malhotra, A. (2005). Diagnosis of ectopic gastric mucosa using $99 \mathrm{Tcm}$ - pertechnetate: Spectrum of scintigraphic findings. British Journal of Radiology, 78(932), 714-720.

13. Sokol, H., Seksik, P., Wendum, D., Bellanger, J., Parc, Y., Cosnes, J., \&Beaugerie, L. (2009). Gastrointestinal bleeding diagnosed using video capsule endoscopy. Gut, 58(9), 2008-2009. https://doi.org/10.1136/ gut.2008.172155

14. Bhattacharya, A., Samujh, R., Rao, K. L. N., \& Mittal, B. R. (2013). Long segment jejuno-ileal duplication cyst with ectopic gastric mucosa detected on 99mTc-pertechnetate scintigraphy. Indian Journal of Nuclear Medicine, 28(2), 96-98. https://doi.org/10.4103/0972-3919.118260 
Guarino et al., Journal of Histology \& Histopathology 2021,

15. Dinarvand, P., Vareedayah, A. A., Phillips, N. J., Hachem, C., \& Lai, J.

(2017). Gastric heterotopia in rectum: A literature review and its diagnostic pitfall. SAGE Open Medical Case Reports, 5, 2050313X1769396.

https://doi.org/10.1177/2050313×17693968

\section{Citation:}

Vaterlini EM, Osio C, Panagini G, Grotto S, Pelosi G, Ferretti A, Luca MD, Marta T, Marenco D, Ceriani V and Guarino S. Exstensive gastric heterotopia of the distal ileum: A case of bowel obstruction. J Histol Histopathol. 2021; 8:3. http://dx.doi.org/10.7243/2055-091X-8-3 